\title{
A FRAMEWORK TO CREATE A VIRTUAL ORGANISATION BREEDING ENVIRONMENT IN THE MOULD AND DIE SECTOR
}

\author{
Rolando Vargas Vallejos ${ }^{1}$, Celson Lima ${ }^{2}$, Gregório Varvakis ${ }^{3}$ \\ ${ }^{1}$ University of Caxias do Sul, BRAZIL (rvvallej@ucs.br) \\ ${ }^{2}$ Centre Scientifique et Technique du Bâtiment (CSTB), FRANCE (c.lima@cstb.fr) \\ ${ }^{3}$ Federal University of Santa Catarina,BRAZIL (grego@deps.ufsc.br)
}

\begin{abstract}
The effective creation of Virtual Enterprises emerges of a previous work and environment called recently as Virtual Organisations Breeding Environment. The framework here proposed is one of the results of five years research project in the field of Collaborative Network Organisations. This paper describes a framework to create and support the Virtual Enterprises life cycle in the mould and die sector. The framework called AmbianCE was validated in a Brazilian Virtual Organisation Breeding Environment.
\end{abstract}

\section{INTRODUCTION}

The Virtual Enterprises (VEs) paradigm has been moved from the research domain to the business application, supported by the recent developments in the Information and Communication Technologies (ICT) and the new approaches in the management field. Dynamic collaborative networks provide intuitive approaches to face the challenges of turbulent markets. A key question is however how to guarantee the basic requirements to enable such collaboration. Afsarmanesh and Camarinha-Matos (2005) affirmed that the formation of any collaborative coalition depends on its members sharing some common goals, possessing some level of mutual trust, having established common (interoperable) infrastructures, and having agreed on some common (business) practices and values.

In order to support the VEs life cycle it is necessary that potential partners will be prepared to participate in such collaboration. It means that these enterprises need a common interoperable infrastructure, common operating rules and cooperation agreements, and principally 'trust' among other aspects. Therefore, the concept of breeding environment has emerged as the necessary context for the effective creation of VEs. Shortly, Virtual organisation Breeding Environment (VBE) is an association of organisations and their related supporting institutions, adhering to a base long term cooperation agreement, and adoption of common operating principles and infrastructures, with the main goal of increasing both their chances 
and their preparedness towards collaboration in potential Virtual Organisations (Afsarmanesh \& Camarinha-Matos, 2005).

This paper describes a framework to support the VE life cycle relying on a VBE. The framework proposed here has been validated in a Brazilian VBE called Virfebras. A peculiar characteristic of Virfebras is that the members are competitors operating in the mould and die sector. One important element in this framework was the application of the theory of Knowledge Management (KM) in order to maximise the capitalisation and dissemination of the knowledge created during a VE life cycle. In the mould and die sector, relevant knowledge is created, especially tacit knowledge. The idea is to transform it into explicit knowledge and store it in different but strongly related layers, which have to be managed properly.

\section{THE VIRFEBRAS VBE}

Virfebras is a VBE that resulted from the partnership involving the following organisations: (i) the University of Caxias do Sul (UCS); (ii) nine mould and die makers; (iii) the Brazilian agency supporting SMEs (SEBRAE-RS); and (iv) the government of the State of Rio Grande do Sul. These enterprises, which have common market interests, decided to take part in a research project coordinated by UCS aiming to learn how to build a cooperative environment supported by ICT.

Virfebras is located in the city of Caxias do Sul, south of Brazil. Whenever an order is submitted to the group, a VE is created. Companies belonging to this VE play two kinds of roles: coordinator (VE-C) and members (VE-M). The VE-C takes responsibility on both technical and legal aspects of the order. When the mould(s) and/or die(s) are delivered to the customer, and there are no more issues to be handled, the VE is dissolved (Vallejos, 2005).

It was necessary five years to create the Virfebras VBE. Only after having structured the VBE was possible to create VEs. Nowadays, within the VBE, several VEs may exist at the same time. It is worth noticing that every company keeps its identity, meaning its own capacity of make business individually.

The Virfebras VBE creation passed through several phases, which will be described in the next sections.

\section{FRAMEWORK TO CREATE A VBE IN THE MOULD AND DIE SECTOR}

Companies aiming at working collaboratively to create VEs should previously develop some competencies and create a favourable environment. For example, it is necessary to develop/deploy a common ICT-based infrastructure to allow the integration/interoperation of the companies among themselves and to develop some additional abilities to adapt the traditional culture of work of both entrepreneurs and employees towards the use of VE/VBE concepts.

The AmbianCE (Ambiente de Criação de Empresas Virtuais) is a framework to support the creation of a VBE tailored to the mould and die sector. It was also developed to face difficulties and barriers that appear when enterprises decided to 
create VEs. AmbianCE provides an environment where trust, competence, and the use of ICT are fundamental in order to support collaborative work.

The lack of trust is one of the problems found when creating VEs. It is overcome with the establishment of long-term relationships amongst mould and die makers. However, the creation of trust is a process built slowly along the time and is solely based on actions and results. The AmbianCE framework makes possible the VBE establishment that permits a long-term relationship among entrepreneurs and employees and creates opportunities for improvement.

The AmbianCE framework (figure 1) is composed of three steps, namely Prepare AmbianCE, Structure AmbianCE, and Act. In addition to that, its deployment is guided by the so-named AmbianCE Strategy, which is based on a KM Program and a Benchmarking Methodology. Such strategy also uses a 'vertical' element called AmbianCE Evolution Vector connecting steps two and three, creating a cyclical process that allows the constant improvement of the framework.

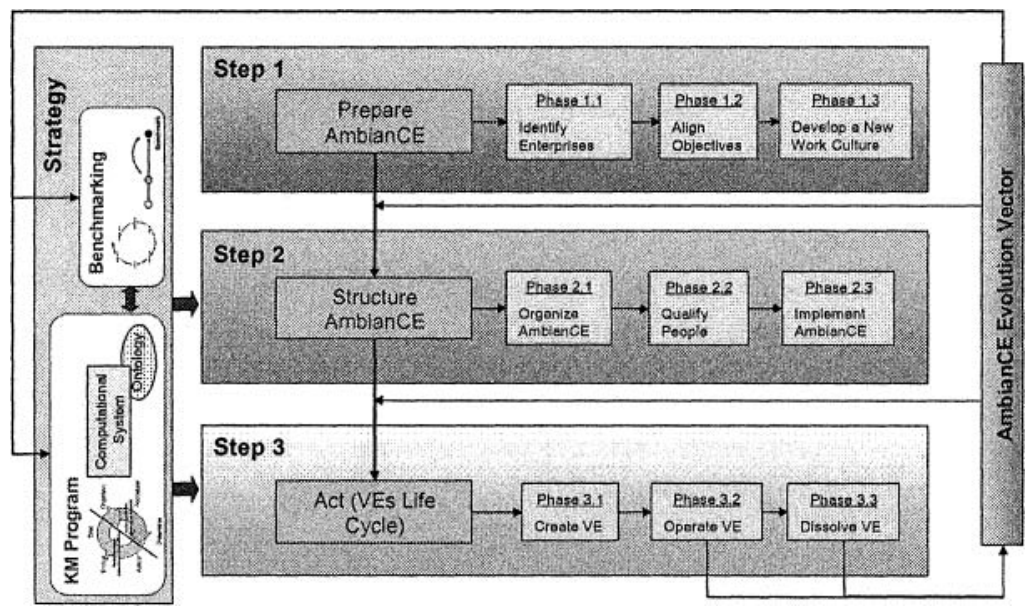

Figure 1 - General overview of the AmbianCE framework

\subsection{Step 1 - Prepare AmbianCE}

This step prepares the necessary environment to create a VBE. It is subdivided in three phases, namely Identify Enterprises, Align Objectives, and Develop a New Work Culture.

\subsubsection{Phase 1.1 - Identify Enterprises}

It aims at identifying the organisations that intend to work in a collaborative form creating VEs. Those organisations could belong to the same industrial districts, clusters, or can be geographically dispersed. If there is no initiative to work collaboratively, it will be necessary to look for industrial districts and clusters (already formed or on the way) where the candidate organisations are operating.

After detecting the organisations with the required potential to create a VBE, it is recommended to conduct interviews with the managers of those organizations, in order to understand their histories, culture of work, partnerships, the existing 
collaboration forms, as well as the willingness to work collaboratively through VEs. It is necessary to organise workshops about VEs and VBEs involving the participation of industrials, universities, technological centres, governmental agencies, and other organisations that support the referred sector.

It is recommended that neutral entities (e.g. universities, research institutes, associations, government, etc.) may coordinate the VBE creation. The authors argue that a VBE formed with competitors could be a good alternative to face the actual turbulent market. At first glance this seems to be a contradiction since a VE is formed with complementary enterprises. However, if well coordinated and created, a VE formed with competitors, with complementary competencies, can have more advantages than disadvantages. The organisations that work in the mould and die sector have similar types of products, same market interests, same problems to access new technologies, difficulties to find prepared employees, and sometimes even if they have similar processes they did not have the same resources (infrastructure, machining machines, CAD/CAE/CAM systems, etc.), etc..

Actually, a lot of products need a set of moulds and/or dies to be manufactured. Generally, one single enterprise cannot manufacture all these tools because of its work capacity. For that reason it is interesting to work in a collaborative way involving more enterprises, even if they are competitors. If the objectives of the collaborative work are well defined, the combination of similarities and differences create more opportunities for the organisations to find solutions and to work as VEs.

\subsubsection{Phase 1.2 - Align Objectives}

In this phase, all organisations that decided to work together should define common objectives to guide their future activities. They must define, at least, one common objective that is fundamental for the success of structuring and operating the VBE. A way to find those objectives is to offer bigger challenges to the members of the VBE, such as the existence of a more reachable market.

It is recommended to organise workshops, seminars and training courses, prepared by experts, in order to identify opportunities and strategies to attend the market. It is vital to emphasize that the union of enterprises will allow to: (i) access new and larger business opportunities; (ii) attend new customers and new markets; and (iii) access new technology and researches. It is suggested to organise visits to potentials customers and to organise trips (or business missions) to analyse new markets. In those trips it will also be possible to work behavioural aspects among entrepreneurs, mainly the aspect of 'trust'.

\subsubsection{Phase 1.3 - Develop a New Work Culture}

It aims to build a new culture of work amongst the entrepreneurs that have the intention to create VEs. The approach used for the construction of that new work culture considers three aspects, namely: Culture of Trust, Competence Culture, and ICT Culture.

The Culture of Trust is considered the most important for the success of VEs. The creation of a VBE is necessary to built trust among the entrepreneurs. In a VBE it is possible to organise courses and activities on organisational behavioural development. Another important and effective activity is to organise visits to each 
VBE's member. Initially the entrepreneur to be visited may fear receiving competitors, but during the visit, he will likely notice that the information exchanged with other entrepreneurs can be very rich. Finally, it is important to organise business travels in order to visit national and international fairs and to visit research centres. In these trips, besides the establishment of contacts with potentials customers, the access to new technologies and the investigation of future markets, the entrepreneurs have the opportunity to develop trust knowing better each other.

The Competence Culture deals with the development, the investment and the consolidation of the organisation competencies. If one enterprise has the intention to participate in a VE, it is mandatory to have at least one competence that differentiates from the others. In a VBE is possible to identify, to develop and to evidence core competencies of each mould and die makers. Knowing the core competencies of the VBE enterprises, it is possible to create efficient VEs and to detect the absence of other core competencies necessary for the VBE.

The ICT Culture targets the correct use of ICT tools/technologies for communication and management of the information exchanged between enterprises. Some mould and die entrepreneurs have personal barriers to use ICT tools. For those entrepreneurs it is necessary to show the benefits and the need of using ICT to participate in VEs. It is necessary to establish patterns for the ICT among enterprises considering several application levels.

\subsection{Step 2 - Structure AmbianCE}

After the preparation of the AmbianCE it is necessary to structure the VBE promoting the establishment of a long-term relationship among mould and die makers. The step Structure AmbianCE is divided in three phases called: Organise AmbianCE, Qualify People and Implement AmbianCE.

\subsubsection{Phase 2.1 - Organise AmbianCE}

It aims to create an organisational and functional structure of the VBE. A hierarchical management structure will guarantee the good operation of the VBE. At least, it is necessary to have a President and a Broker to instantiate the VBE operation. Other actors and functions can appear according to the entrepreneurs' expectations and needs. The President has the responsibility to coordinate the creation of the statute, the ethics code, operational procedures, etc.. The Broker has the responsibility to detect business opportunities, to establish contacts with potential customers, to identify needs of core competencies, etc..

The VBE statute, the ethics code and the operational procedures should be created. The statute should cover, for instance, the admittance of new enterprises, the partners' responsibilities, etc.. The ethics code rules behavioural aspects, the entrepreneurs' bonuses, the entrepreneurs' punishments, etc.. The operational procedures rule the dynamics of the VEs life cycle. It is necessary be define clearly the functions and responsibilities of the VE Coordinator (VE-C) and VE Members (VE-Ms). For example, the VE-C should guarantee the execution of the Distributed Business Process (DBP) and to assume the technical and administrative responsibility of the order being executed. In a VBE, several VEs may exist simultaneously, and each organisation can play both roles in different EVs, at the 
same time. The operational procedures should consider the criteria to define who is who (i.e., who is the VE-C and who are the VE-Ms).

\subsubsection{Phase 2.2-Qualify People}

In this phase the entrepreneurs that understood the importance to work collaboratively through VEs, have the challenge to involve their employees in that kind of collaborative work.

Some actions that the entrepreneurs should organise in their own enterprises are workshops and meetings with their employees to understand the benefits and the challenges of working creating VEs. It is recommended to form work groups to analyze and optimize different Business Processes (BPs). Those work groups should establish metrics to accomplish their performance along the time. It is recommended to organise meetings with the participation of several work groups of different enterprises. In those meetings each work group could present the improvements obtained. It is possible to organise visits of the work groups in different mould and die enterprises to show practically the improvements in that BPs. These actions are fundamental to develop a new work culture in employees involved in a VBE. The employees should feel that the new collaborative work brings benefits and opportunities, besides improving their work conditions.

\subsubsection{Phase 2.3-Implement AmbianCE}

To implement AmbianCE is necessary to know all the core competencies of each enterprise and to identify the fundamental BPs to create and operate efficient VEs in mould and die sector. It is important to establish metrics for each fundamental BP.

To identify fundamental BPs and to accomplish their performance along the time it is suggested the application of a Benchmarking methodology. In the present work a Benchmarking methodology for mould and die enterprises was developed. The Benchmarking methodology is to be applied in a cyclical way and is being considered as a fundamental element to implement AmbianCE.

Other element that is fundamental to structure the VBE successfully is the KM application. This work proposes the implementation of a KM Program as a strategy of the AmbianCE framework aiming to reuse and disseminate knowledge. The implementation of a KM Program should be one of the primary actions of the VBE in formation. The effectiveness and the efficiency of future VEs depend on the good management of the knowledge created within the VBE.

In order to validate the operational procedures (ruling the VBE) to create, operate and dissolve VEs, it is suggested to look for 'pilot' business opportunities. It is necessary to evaluate these procedures, agreements, contracts, infrastructure and results. It is necessary to involve all VBE members to discuss these aspects. After having performed the 'pilot' experiences and solved (likely) technical, operational and behavioural problems the VBE is considered ready to attend new business opportunities.

\subsection{Step 3- Act}

The Step 3 of the AmbianCE framework treats about the VEs life cycle and it is divided into three phases, namely Create VE, Operate VE, and Dissolve VE. 


\subsubsection{Phase 3.1 - Create VE}

This phase targets the creation of the most efficient and competitive VE to attend a given business opportunity. When a business opportunity appears the VBE procedures to create a VE should be followed in order to select VE-C and VE-Ms. It should be defined precisely the DBP, considering their BPs delivered time. Important is to discuss the responsibilities and to establish clearly the profits and the risks that each company will assume.

Another issue to be discussed during the VE creation is the management of information (of the different enterprises) to be shared along the BPs. The efficiency of a VE depends on information sharing, including those considered as 'private' for a certain enterprise. How to treat this 'confidential' information and knowledge during the VE life cycle?

Once discussed the issues above mentioned, it is necessary to sign agreements and contracts between the enterprises that form the VE. Mould and die makers should create the habit of formalise their collaboration agreements in order to protect themselves from any problems that may occur in the future. After documenting these agreements and contracts the VE is considered created.

\subsubsection{Phase 3.2 - Operate VE}

In this phase the VE executes the customer order, performing the different BPs efficiently. It is important to monitor and to record the BPs, which could be done through a performance evaluation during the execution of BPs.

The information exchange is intensive, not only inside of each enterprise, but also among the VE partners. The information should be treated in several levels and should be separated in two groups: 'private' and 'public'.

Unexpected problems can appear along the BPs execution, which should be solved following the VBE norms and procedures. In extreme cases the VE can be reconfigured, to be treated in the AmbianCE Evolution Vector.

\subsubsection{Phase 3.3 - Dissolve VE}

Once concluded the DBP and having given the product to the customer, the VE should be dissolved. It is suggest to develop questionnaires to evaluate the customer, the VE-C and the VE-Ms satisfaction.

It should be capitalized the new information acquired along the BPs. The information to be reused should be stored and disseminated for the right people in the VBE. It should be evaluated if the VE will be created again in the future or not, the execution of the agreements and the contracts, as well as the VBE norms and the procedures.

\subsection{AmbianCE Strategy}

The base of the AmbianCE Strategy is the application of a KM Program supported by a Benchmarking Methodology. The AmbianCE Strategy has an integration vector 
called AmbianCE Evolution Vector and is supported by the AmbianCE computational system and a Mould and die Ontology.

\subsubsection{KM Program}

The implementation of a KM program should be one of the priority actions of the VBE. The effectiveness of a VE is risked when the information along the DBP is not managed appropriately. In addition to the information, knowledge is created and applied constantly during the VE life cycle. This knowledge should be stored and disseminated aiming to be reused in future BPs and in the future VEs creation. The effectiveness of future VEs depends on a good knowledge management.

This work adopted and adapted the KM model proposed by Nissen et al. (2000), which is composed by six phases, namely Create, Organise, Formalize, Distribute, Apply, and Evolve.

Create is the phase in which is necessary to invest to grow up (within the VBE) the conditions required to allow people to create new knowledge. In this phase the Nonaka and Takeuchi (1997) KM model and the Hutchins (2000) approach of Distributed Cognition are considered.

Organise deals with the knowledge organisation. The capture of knowledge can happen searching and finding existing knowledge or can start from the creation of new knowledge. To seek existing knowledge it can be used, for instance, 'knowledge map systems', 'semantic networks', 'knowledge-based systems', etc.. Once captured the desired knowledge it must be organised following some rules, previously defined to facilitate its classification, storage, dissemination and future capitalisation. The knowledge organisation can be appropriately driven with a specific ontology.

Formalise is the phase the knowledge should be validated monitoring its use from other experts. It should also guarantee that knowledge is rightly updated, through the creation of new knowledge and the exclusion of obsolete knowledge.

Distribute means to disseminate the knowledge for the right people considering previous criterion in an automatic way, which means that work groups should be created with common interests.

Apply is the phase in which the knowledge is capitalised meaning that it is applied in real situations into the DBP producing concrete benefits. To evaluate the results after the knowledge use it is suggested the establishment of metrics.

The AmbianCE Evolution Vector integrates the KM model with the AmbianCE Strategy in order to create a continuous life cycle.

\subsubsection{Benchmarking Methodology}

It was developed a Benchmarking methodology to implement the KM program in a mould and die VBE that was presented in (Vallejos \& Gomes, 2004). Benchmarking is a continuous and systematic process used to evaluate enterprises' BPs in order to establish referential patterns. In addition, with the application of the Benchmarking methodology, a behavioural change occurs in the employees when they recognise the differences of BPs in other companies.

\subsubsection{AmbianCE Evolution Vector}


The AmbianCE Evolution Vector integrates Steps 2 and 3, Structure AmbianCE and Act (VEs life cycle) with the Benchmarking methodology and consequently with the $\mathrm{KM}$ program. The objective of this vector is to create a cyclical and continuous dynamics in the AmbianCE framework. In this phase it is possible to reconfigure a $\mathrm{VE}$ in process and to optimize the creation of future VEs using the KM approach. It can also identify the need of new enterprises with specific core competencies, or to decide for the VBE qualification contracting consultancies and research projects.

\subsubsection{AmbianCE Computational System}

The use of a KM computational system to support the AmbianCE Strategy is necessary. In the present work it was adopted the e-COGNOS project approach to support the $\mathrm{KM}$ in the Building and Construction industry (http//:www.ecognos.org). The e-COGNOS approach is based on the development of the $e$ COGNOS Knowledge Management Infrastructure (e-CKMI). The AmbianCE Computational System used the e-CKMI methodology for their development. This system can be described differentiating its architecture, functionality and technical characteristics.

Architecture characteristics: Web-centred environment, ontology-based, push mechanisms, autonomous processes, and knowledge layer interdependence

Functional characteristics: human-centred, easy to use, appealing, adaptive, configurable, based on experiences and open.

Technical characteristics: interoperability, integration, flexibility, scalability, sustainability, heterogeneous inter-related knowledge sources, and large knowledge sources.

More details of the AmbianCE Computational System were published in (Lima \& Vallejos, 2002).

\subsubsection{Mould and Die Ontology}

To support the AmbianCE Computational System was necessary to develop a mould and die ontology. According to Lima et al. (2002) the ontology allows the semantics process and communication of different sources of information between different people and computational systems. Ontology guarantees the no existence of semantic ambiguity on knowledge shared in a certain work domain. In the present work it was not intended to provide the ultimate and definitive ontology for the mould and die sector, but it can serve as a base for future developments.

\section{CONCLUSION}

This paper presented AmbianCE, a framework to create a VBE in the mould and die sector. The proposal is to develop a VBE before the VEs creation aiming to prepare mould and die makers for this kind of collaborative work. The VBE allows them to develop a new culture of work and to prepare an appropriate infrastructure for their operation. 
The AmbianCE framework is constituted of three steps (Prepare AmbianCE, Structure AmbianCE, Act) and has a Strategy which is based on a KM Program and a Benchmarking Methodology for his implementation.

The verification of the applicability of the present framework was realized in a VBE called Virfebras. The construction of the VBE was possible in five years of work, creating a basic infrastructure, an organizational structure, norms and operational procedures and, mainly, a new work culture. AmbianCE facilitates the development of a new work culture for the organizations considering aspects of trust, core competencies and the use of ICT.

As result it was possible to create a favorable environment to launch the VEs life cycle. It should be pointed out that even the Virfebras members are competitors; they create efficient and competitive VEs aiming at new customers and markets. In the beginning of the work it was common to observe that entrepreneurs and employees used to hide information from the partners. However, they realised that this behaviour should be replaced by a new one more suitable for a collaborative environment. They started sharing information and learned that they usually have very similar problems. If they started sharing solutions, every enterprise could benefit from this exchange process. The Virfebras enterprises have grew in infrastructure, contract more employees, developed new customers and suppliers and, mainly, adopted a new collaborative form of work.

Due the obtained results, the proposed framework shows to be efficient turning future VEs more competitive. If well implemented, the KM Program could be considered as a important facilitator factor for the VBE formation, motivating other enterprises to adopt that kind of collaboration work.

\section{REFERENCES}

1. Afsarmanesh, H.; Camarinha-Matos, L.M. A framework for management of Virtual Organization Breeding Environments. In: Camarinha-Matos, L. M.; Afsarmanesh, H.; Ortiz A. Collaborative Networks and their Breeding Environments. IFIP Vol. 186. Springer, 2005.

2. Camarinha-Matos, L. M.; Afsarmanesh H. The virtual enterprise concept. In: Infrastructures for Virtual Enterprises - Networking Industrial Enterprises. Kluwer Academic Publishers, 1999.

3. Hutchins, E. Distributed cognition. International Encyclopedia of the Social and Behavioral Science. IESBS Distributed Cognition. Lhtp://www iesbscom/pds . Accessed in: 18 th may, 2000.

4. Lima, C. P.; Vallejos, R. V. Towards a Knowledge Management infrastructure to support Virtual Enterprises. In: The European Conference on Information and Communication Technology Advances and Innovation in the Knowledge Society (eSM@ART 2002), Salford. Towards a European Knowledge Economy in the Construction and Related Sectors. Salford University, 2002.

5. Lima, C. P. et al. The e-CKMI: an Ontology-enabled platform to support KM in construction. In: eWork 2002 Conference. Prague; oct. 2002.

6. Nissen, M.; Kamel, M.; Sengupta, K. Integrated Analysis and Design of Knowledge Systems and Processes. In: Malhotra, Y. Knowledge Management and Virtual Organizations. UK: Idea Group Publishing, 2000.

7. Nonaka, I.; Takeuchi, H. Criação de Conhecimento na Empresa: Como as empresas japonesas geram a dinâmica da inovação. 6. ed. Rio de Janeiro: Campus, 1997.

8. Vallejos R.V. Um modelo para formação de Empresas Virtuais no setor de moldes e matrizes. Tese de doutorado. Universidade Federal de Santa Catarina, 2005.

9. Vallejos, R. V.; Gomes, J. O. Applying a Benchmarking methodology to empower a Virtual Organization. In: $6^{\text {th }}$ International Conference on Information Technology for Balanced Automation Systems in Manufacturing and Services (BASYS 2004), Vienna. Emerging Solutions for Future Manufacturing Systems. Springer, 2004. 